\title{
A Review Characterization of Some Material Oxides using Ultrasonic Techniques
}

\author{
Mohini Kuldevi \\ M. Tech \\ Bundelkhand University \\ Jhansi, Uttar Pradesh, India \\ mohinikuldevi@gmail.com
}

\author{
Dr. Sanjeev Kumar Srivastava \\ Professor \\ Bundelkhand University \\ Jhansi, Uttar Pradesh, India
}

\begin{abstract}
Several technological advances have begun in the industry today. With more advances comes a greater risk of errors. Ultrasound techniques are one of the best known methods for detecting defects and increasing efficiency. There are many variations of this technology and each has its own application depending on the needs of the situation. This paper provides an overview of general ultrasound technology and also describes the characterization of materials using ultrasound testing.
\end{abstract}

Keywords: Ultrasonic, Graphite Nodularity, Sound Velocity, Attenuation

\section{INTRODUCTION}

Nanotechnology is the basis for improving knowledge of materials and phenomena at the nanoscale. This is essential for the development of a device. Since the sensors are capable of recording chemical information from the environment in real time, they are arousing great interest and will therefore have an increasing influence on daily life. Chemical sensors can detect toxic and explosive analytes, be integrated into safety systems to protect workers from chemical hazards, be used to monitor the environment, health and wealth, and to control the food chain. Much effort has been made in recent years to improve the quality and safety of the food chain, as ingesting food that has not been properly stored or handled is one of the most common reasons for hospitalization. Chemical sensors can play a crucial role in all of these applications. Metal oxides were the first to be marketed as thick film conductometric chemical sensors and remain the most promising materials for chemical detection. Chemical metal oxide sensors are more stable and reproducible than organic sensors. However, to be used in real cases, these devices must meet many requirements such as: High detection performance in terms of sensitivity, selectivity, reaction kinetics and reliability the design of active materials is essential and must be the starting point for the control of the functional parameters of the terminal. However, great attention must be paid to integrating the active material into the transducer. In order to have a stable chemical sensor, not only the active material but all the components of the device, such as B. the electrical contacts and the heating system, are stable and reliable. Graphene, a two-dimensional material, has recently gained a lot of attention due to its various properties such as electronic, mechanical and thermal properties [1].

These unique properties of graphene make it a suitable candidate for many applications such as nano electronics, nanocomposites, and super capacitors, chemical and biological sensors [2]. Graphene nanosheets have been produced by various approaches such as micromechanical cleavage, epitaxy, chemical and thermal reduction of graphene oxide (GO) [3]. Compared to the other methods mentioned above, the chemical reduction of exfoliated GO is the most promising due to the inexpensive large-scale production of graphene [4]. GO can be produced by oxidation of graphite with many available methods such as the Brodie method, the Hummer method or the Staudenmaier method [5]. Like graphite, GO also has a layered structure that contains oxidized graphene layers (or "GO layers"), the base planes of which have epoxy and hydroxyl groups. Carbonyl and carboxy groups at the edges [5]. These oxygen features of GO make it more hydrophilic and water molecules can easily intercalate in the interlayer [6]. The different physical, chemical, optical and electronic properties of graphene nanosheets strongly depend on their size and structure, which are influenced by the synthetic pathways. [7].

GO can be reduced to graphene using suitable chemical reducing agents such as hydrazine hydrate, sodium borohydrate and biological reducing agents such as glucose, ascorbic acid, galactose, etc. [8] [9]. Various approaches such as chemical reduction, hydrothermal method, Solvo thermal method and microwave irradiation method have been used for the reduction of GO in graphene layers [10] [11] [12] [13]. Although there are many synthetic pathways available for reducing GO to graphene, a unique method for synthesizing high-quality graphene sheets using inexpensive technology is 
not yet known. The important parameter in the GO reduction reaction should be targeted: the restoration of the conjugated $\pi-\pi$ network in graphene and the removal of the oxygencontaining functional groups that are bound to graphene, as they can modify the electronic structure of graphene. Today, chemists follow combined reduction techniques involving two or three steps of the reduction reaction to obtain single-layer graphene sheets [4]. These methods use sophisticated tools, high costs, use of toxic gases and longer reaction times [14]. Therefore, the development of a new synthesis strategy for 2D graph synthesis using inexpensive and soft synthetic pathways is of great importance and should be investigated.

\section{LITERATURE REVIEW}

Sanaz Alamdari et al. [15] In this context, zinc oxide nanoparticles ( $\mathrm{ZnO}-\mathrm{NP})$ were produced from S. ebulus leaf extract and their physicochemical properties studied. The results of X-ray diffraction (XRD) showed that the ZnO NPs produced are highly crystalline and have a wurtzite crystal structure. The average crystallite size of the NPs produced was about $17 \mathrm{~nm}$. NPs synthesized in green showed excellent absorption in the UV range and strong yellow-orange emission at room temperature. The prepared nanoparticles showed good antibacterial activity against various organisms and acceptable photocatalytic degradation of the methylene blue coloring pollutants. The results obtained showed that the biosynthesized NP $\mathrm{ZnO}$ have interesting properties for various possible future applications.

Farazas et al. [16] Graphene oxide (GO) was produced by an improved green chemical process using graphite flakes, $\mathrm{KMnO} 4$, a mixture of $\mathrm{H} 2 \mathrm{SO} 4$ / $\mathrm{H} 3 \mathrm{PO} 4, \mathrm{H} 2 \mathrm{O} 2$ and $\mathrm{HCl}$. Ultrasound was used in several steps to separate the oxidized layers of GO. The use of ultrasound optimizes the action of the reagents and reduces the required quantities of chemicals used. This method produces graphene oxide, which is heavily oxidized with a greater distance between the layers than the Hummers method, without producing toxic gases. The final product was characterized by UV-VIS, XRD, optical microscopy, TEM and Raman spectroscopy and all the techniques used confirmed the desired result. The results showed that this inexpensive method is fully scalable without creating unwanted environmental influences.

Maryam Sabbaghan et al. [17] In this study, an efficient pathway for graphene oxide (GO) and reduced GO (RGO) synthesis was developed using alternately an ultrasound probe and a bath. GERD was produced under acidic and neutral conditions. The products were characterized using FTIR, XRD, Raman spectroscopy, SEM and AFM. In GO, wellpeeled and smooth leaves without crumbs are observed. RGO synthesized in acidic media had fewer layers and a smaller crystallite size than that derived from neutral conditions. Electrochemical studies have shown a greater effective surface area and better electrocatalytic activity for GERD synthesized in acid medium (GERD1). The electrochemical sensing ability of the RGO1-modified glassy carbon electrode (RGO1 / GCE) versus dopamine (DA) was investigated using cyclic and differential pulse voltammetry methods.

M. Gouda et al. [18] This paper deals with the synthesis of some nanometallic oxides by means of microwave irradiation technology and their application to improve the multifunctional properties of cotton fabrics. Cotton fabrics containing nanometallic oxides were produced by thiol modification of cotton fabric samples, then dipped in the precursors of the metal salt solutions and transferred to the microwave oven. The surface morphology and quantitative analysis of the obtained modified cotton fabrics, which contained nanometallic oxides, were examined by scanning electron microscopy in combination with high-energy dispersive X-rays (SEM-EDX). The shape and distribution of nanoscale oxide in tissue samples was analyzed by transmission electron microscopy of tissue samples with a cross section.

Amir Hassanjani-Roshan et al. [19] The iron oxide ( $\alpha$-Fe2O3) nanoparticles were produced using a chemical process. Process parameters such as temperature, sonication time and ultrasound power play an important role in the size and morphology of the finished products. Iron oxide nanoparticles were characterized by transmission electron microscopy, powder X-ray diffraction, as well as differential thermo gravimetric and thermal analyzes. It is estimated from transmission electron microscope observations that the size of the iron oxide nanoparticles is significantly less than $19 \mathrm{~nm}$. The powder X-ray diffraction data after quenching provides direct evidence that iron oxide formed during the chemical process of the wires.

\section{UltRAsONIC TESTING}

The Ultrasound Test Evaluation (UT) System consists of a transmitter and receiver circuit, a conversion tool, and display devices. Based on the information transmitted by the signal, it was possible to obtain the position of the crack, the size of the defect, the orientation and other properties [20]. The benefits of ultrasonic testing include speed of analysis, good resolution and fault detection capability, and the ability to be used in the field. Disadvantages include configuration difficulty, the skills required to accurately scan a part, and the need for a test pattern to ensure accurate testing. This type of testing is ideal for use on assembly lines where the same part design needs to be tested multiple times. There are two approaches to ultrasonic NDT which are generally used in different applications. Echo of impulses and transmission approaches. Both approaches use high-frequency sound waves of the order of 1 to $50 \mathrm{MHz}$ to detect internal defects in a material [21]. 
The ultrasound test can be performed in three modes: transmission, reflection and backscatter. Each of them uses a range of transducers, coupling media and frequencies. The pulsed echo ultrasound method facilitates the detection of defects in homogeneous materials. With this method, the operator is more concerned about shaft run time and energy loss due to shaft attenuation and dispersion due to errors. It allows you to identify inconsistencies in a material, be it homogeneous or heterogeneous. Ultrasonic pulse rate measurements are suitable for the detection, localization and imaging of large defects, as well as for quality control [22]. The ultrasonic permeability method differs from conventional ultrasonic methods. This method keeps the transducer and receiver away from the surface and at a fixed distance from the sample. This is particularly advantageous when complex geometries do not allow a conventional transducer and receiver to contact the surface of the part. The most commonly used property indicators are wave propagation speed and loss of amplitude (or energy). Some of the test methods described here only deal with one property, while others, more versatile, can measure two or three [23]. Most applications only take into account the pulse rate and relate it to various parameters. Considering the loss of energy, it is possible to discover some additional properties of a material [24]. Several authors have examined the impulse damping analysis method [25]. Diffusion, absorption and geometry are three parameters that affect attenuation. Small discontinuities such as grain boundaries are the cause of the diffusion.

\section{MATERIAl CharaCterization By Ultrasonic} TESTING

\section{A. Velocity}

The speed of ultrasound in longitudinal and cutting modes can be related to various material properties. The measurement includes determining the transit time between the first and second reflections from the back surface and dividing it by the distance traveled by the ultrasound. The accuracy of these measurements depends on the accuracy with which the travel time and thickness of the components are measured. In cases where only the surface of the component is accessible (in situ applications), the speed ratio (longitudinal shear to shear) serves as a useful parameter. Unlike speed determination, where component thickness is required as an input, the speed ratio can only be determined from the time path data for longitudinal and shear waves. Some of the techniques used to determine the velocity are the impulse echo superposition technique, the impulse superposition method, the sing-around method and the phase comparison method [40]. With advances in electronics and digital technology, speed can be measured with an accuracy of less than $1 \mathrm{~m} / \mathrm{s}$, making it a very reliable parameter for characterizing material properties.

\section{B. Attenuation}

Attenuation refers to the loss of sound energy as the ultrasonic beam passes through the material. Attenuation consists of two components: absorption and diffusion. The loss of energy by absorption is the result of mechanisms such as dislocation damping, hysteresis losses, thermoelastic effects, etc. The diffusion loss in polycrystalline materials depends on the ratio between the particle size (D) and the wavelength $(\lambda)$. There are three different scattering regimes, each with different damping coefficients depending on the grain size (D), wavelength $(\lambda)$ and frequency ( $\mathrm{f}$ ). The attenuation coefficient is measured by finding the difference in amplitude $(\mathrm{dB})$ between the two back surface signals, then dividing it by the total distance traveled. It is expressed in $\mathrm{dB} / \mathrm{mm}$. In more advanced techniques, the frequency spectrum (frequency-amplitude diagram) is obtained from two consecutive signals from the back surface. The attenuation coefficient at different frequencies is determined from these spectra. The attenuation measurement technique is often used in the characterization of material properties to determine the particle size, the distribution of the second phase particles such as inclusions and porosities, the distribution of diffuse discontinuities such as microcracks, etc.

\section{Backscatter amplitude}

Backscattered signals (often referred to as noise) from grain boundaries or second-stage particles pose many problems during ultrasonic inspection for fault detection. However, broadcast signals contain information on the size and type of transmission. Techniques have been established to quantitatively determine the backscatter amplitude to assess the grain size and particle distribution of the second phase. Backscatter measurement is also used to determine diffuse discontinuities such as microcracks that occur in components due to various degradation mechanisms.

\section{Spectral analysis}

The ultrasonic wave traveling through the material is made up of many frequency components. The energy distribution (amplitude) at different frequencies (frequency spectrum) can be obtained by performing the fast Fourier transform of the back surface reflection. Two frequency spectrum parameters that can be useful for material characterization studies are peak frequency and bandwidth. Due to changes in material properties, some of the frequency components are preferably attenuated over others. This leads to a change in the spectrum, which can be related to the change in the properties of the material.

\section{E. Critical angles}

When the longitudinal wave traveling through one medium is incident at an angle on the interface with the other medium, it undergoes refraction and mode conversion. The angle of refraction of the longitudinal and shear waves in another 
medium depends on the angle of incidence and the speed of the sound ratio in the two mediums (Snell's law). An increase in the angle of incidence leads to an increase in the fractured angles of the longitudinal and shear waves. At the first critical angle, the refracted longitudinal wave travels along the surface and leaves only a shear wave in the other medium. At the second critical angle, the fractured cut begins to move along the surface. The values of these critical angles are very sensitive to the surface properties of the material. These can be measured very accurately by examining the change in surface properties and housing depth measurements in components with the protractor.

\section{F. Acoustic microscopy}

Acoustic microscopy refers to high-resolution, high-frequency ultrasonic inspection techniques that create images of features beneath the sample's surface. Unlike light microscopy, where only information on surface characteristics can be obtained, acoustic microscopes provide information on volume or volume characteristics. With conventional ultrasound tests, the test frequency varies between 1 and $10 \mathrm{MHz}$. Frequencies up to $1 \mathrm{GHz}$ or higher are used in acoustic microscopy. At these frequencies, the wavelength in most engineering materials is on the order of a few micrometers. Therefore, acoustic microscopes achieve resolution of the order of magnitude obtained with optical microscopes.

\section{Acousto-Ultrasonic (Au)}

A non-destructive evaluation technique suitable for rapid part inspection is Acousto-Ultrasonic (AU). This seems particularly interesting and can be the one-stop solution for thick and geometrically complex parts or structures, as well as for high-volume industrial applications that require rapid part production. A series of ultrasonic waveforms are emitted and detected using a configuration of multiple probes surrounding the structure. A probe cradle assembly can be used for fabricated parts or for on-site inspection. However, there is great potential in RTM process control, where probes can be integrated immediately into the mold. Digital signal processing (fast Fourier transform) is used to extract the relevant waveform discriminators, and pattern recognition theory is then applied to rank their respective characteristics based on the results obtained from a set. Learning signals obtained from a selection of "good" and "bad" "structures. During an Acousto-Ultasonic test cycle, the externally generated stress waves propagate through the room interacting with all the different components of the material. Since AU is a volumetric test, the data generated contains information about the entire part Multi-probe configuration requires optimization with wave propagation modeling. Transducer emitter / emitter couplings typically include classic probe configurations of the same side, opposite and cross section.

\section{CONCLUSION}

This paper looked at ultrasound and acousto-sonographic (AU) examinations. This article describes attenuation, critical angles, acoustic microscopy. Factors such as efficacy and safety should be used to analyze the best method to use. Furthermore, the method chosen must minimize the costs incurred during operation. It is based on methods that depend on the use of physical values to determine the properties of materials. Ultrasound techniques are sensitive to changes in graphite morphology and can therefore also be used to predict mechanical properties.

\section{REFERENCES}

[1] M.C. Wu, J.S. Corneille, C.A. Estrada, J.W. He, D.W. Goodman, Chem. Phys. Lett. 182 (1991) 472

[2] S.K. Shukla, G.K. Parashar, A.P. Mishra, P. Misra, B.C. Yadav, R.K. Shukla, L.M. Bali, G.C. Dubey, Sens. Actuators B 98 (2004) 5-11.

[3] L.D. Zhang, J.M. Mo, Nanometer Materials, Science Press, Liaoning, 1994, pp. 303-306 (in Chinese).

[4] J. Sawai, H. Kojima, H. Igarashi, A. Hashimoto, S. Shoji, T. Sawaki, A. Hakoda, E. Kawada, T. Kokugan, M. Shimizu, World J. Microb. Biotechnol. 16 (2000) 187.

[5] O. Yamamoto, J. Sawai, T. Sasamoto, Int. J. Inorg. Mater. 2 (2000) 451.

[6] O.B. Koper, J.S. Klabunde, G.L. Marchin, K.J. Klabunde, P. Stoimenov, L. Bohra, Curr. Microbiol. 44 (2002) 49-55.

[7] P.K. Stoimenov, R.L. Klinger, G.L. Marchin, K.J. Klabunde, Langmuir 18 (2002) 6679.

[8] L. Huang, D. Li, Y. Lin, M. Wei, D.G. Evans, X. Duan, J. Inorg. Biochem. 99 (2005) 986.

[9] Y.J. Han, J. Kim, G.D. Stucky, Chem. Mater. 12 (2000) 2068.

[10] J. Hu, T.W. Odom, C.M. Lieber, Acc. Chem. Res. 32 (1999) 435.

[11] C.C. Chen, C.Y. Chao, Z.H. Lang, Chem. Mater. 12 (2000) 1516

[12] M.B. Mohamed, K.Z. Ismail, S. Link, M.A. El-Sayer, J. Phys. Chem. 102 (1998) 9370.

[13] N. Takahashi, Solid State Sci. 9 (2007) 722.

[14] S. Zhang, A.R. Horrocks, Prog. Polym. Sci. 28 (2003) 1517.

[15] Sanaz Alamdari, Morteza Sasani Ghamsari "Preparation and Characterization of Zinc Oxide Nanoparticles Using Leaf Extract of Sambucus ebulus", Appl. Sci. 2020, 10, 3620; doi:10.3390/app10103620.

[16] Farazas, A. Mavropoulos "Ultrasound Assisted Green Synthesis and Characterization of Graphene Oxide", Int. J. Nanosci. Nanotechnol., Vol. 14, No. 1, March. 2018, pp. 11-17.

[17] Maryam Sabbaghan, "Ultrasonic route synthesis, characterization and electrochemical study of graphene oxide and reduced graphene oxide, Research on Chemical Intermediate, 13 october 2018.

[18] M. Gouda, A. Aljaafari "Preparation and Characterization of Some Nanometal Oxides Using Microwave Technique and Their Application to Cotton Fabrics", Journal of Nanomaterials Volume 2015, Article ID 586904, 9 pages.

[19] Amir Hassanjani-Roshan, Mohammad Reza Vaezi "Synthesis and Characterization of Iron Oxide Nanoparticles via Sonochemical method", November 2010, Particuology 9(1).

[20] Lu Y. (2010); "Non-destructive Evaluation on Concrete Materials and Structures using Cement-based Piezoelectric Sensor"; (3457480 Ph.D.), Hong Kong University of Science and Technology (Hong Kong), Ann Arbor. Retrieved from http://search.proquest.com/docview/880396184? accountid=28930 ProQuest Dissertations \& Theses Global database. 
[21] Oguma I., Goto R., Sugiura T. (2012); "Ultrasonic inspection of an internal flaw in a ferromagnetic specimen using angle beam"; EMATs. Przeglad Elektrotechniczny, 88(7B), 78-81.

[22] Ducharne B., Guyomar D., Sébald G., Zhang B. (2015); 10 "Modeling energy losses in power ultrasound transducers"; In J. A. Gallego- Juárez \& K. F. Graff (Eds.), Power Ultrasonics (pp. 241256). Oxford: Woodhead Publishing.

[23] Karabutov A. A., Podymova N. B. (2014a); "Quantitative analysis of the influence of voids and delaminations on acoustic attenuation in CFRP composites by the laser-ultrasonic spectroscopy method Composites Part B: Engineering"; 56(0), 238-244.

[24] El-Sabbagh A., Steuernagel L., Ziegmann G. (2013); "Characterisation of flax polypropylene composites using ultrasonic longitudinal sound wave technique" Composites Part B: Engineering, 45(1), 1164-1172. doi: http://dx.doi.org/10.1016/j.compositesb.2012.06.010

[25] Genovés V., Gosálbez J., Miralles R., Bonilla M., Payá J. (2015); "Ultrasonic characterization of GRC with high percentage of fly ash substitution"; Ultrasonics, 60, 88-95. doi: http://dx.doi.org/10.1016/j.ultras.2015.02.016 